\title{
Transformaciones integrales y sus aplicaciones en finanzas
}

\author{
John Freddy Moreno Trujillo*
}

* Matemático y Magíster en Matemática Aplicada de la Universidad Nacional de Colombia. Docente Investigador de la Facultad de Finanzas, Gobierno y Relaciones Internacionales de la Universidad Externado de Colombia.jhon.moreno@uexternado.edu.co

Fecha recepción: 01 de junio de 2015.

Fecha de aceptación: 01 de agosto de 2015.

Forma de citar

Moreno Trujillo, J.F. (2015). Transformaciones integrales y sus aplicaciones en nanzas. ODEON,

9, pp. 257-265. DOI: http://dx.doi.org/10.18601/17941113.n9.07 



\section{Introducción}

En la teoría y la práctica financiera es bien conocida la importancia de la ecuación diferencial parcial de Black-Scholes (EDP-BS) en la valoración de derivados financieros. Esta ecuación permite encontrar o aproximar la función que establece el valor de un derivado, bajo determinadas condiciones iniciales y de frontera. Si denotamos por $V\left(t, S_{t}\right)$ al precio de un derivado en el instante $t$, mostrando que es función del tiempo $t$ y del precio del subyacente $S_{t}$, la EDP-BS es:

$$
\left\{\begin{array}{l}
\frac{\partial V}{\partial t}+r_{f} S_{t} \frac{\partial V}{\partial S_{t}}+\frac{1}{2} \sigma^{2} S_{t}^{2} \frac{\partial^{2} V}{\partial S_{t}^{2}}-r_{f} V=0 \\
V\left(T, S_{T}\right)=f\left(S_{T}\right)
\end{array}\right.
$$

donde $r_{f}$ es la tasa de interés libre de riesgo y $f\left(S_{T}\right)$ es el valor del derivado en el instante de vencimiento $T$.

Existen diversos métodos para la resolución de esta ecuación o de ecuaciones análogas relacionadas con la valoración de activos contingentes particulares, por ejemplo, procedimientos analíticos vía cambios de variable o métodos numéricos que buscan aproximaciones de las derivadas presentes en la ecuación mediante diferencias finitas.

En este artículo se expone la aproximación a la resolución de esta ecuación mediante la aplicación de transformaciones integrales, en particular las transformadas de Mellin y Laplace. En este procedimiento se aplica una transformación integral sobre la ecuación diferencial parcial considerada, transformándola en una ecuación diferencial ordinaria resoluble por métodos estándar, para luego aplicar sobre la solución encontrada la transformación inversa. En la figura 1 se esquematiza el procedimiento, donde $\mathcal{I}$ denota la transformación integral aplicada e $\mathcal{I}^{-1}$ su transformación inversa. 
Solución

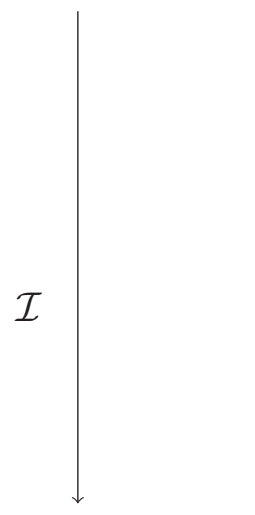

ODE+Condiciones

Solución

Figura 1: Resolución de la EDP por transformación integral.

\section{Transformaciones integrales}

La transformación integral de una función $f(x)$ sobre $[a, b]$, denotada por $\mathcal{I}\{f(x)\}=$ $F(k)$, se define como:

$$
\mathcal{I}\{f(x)\}=F(k)=\int_{a}^{b} K(x, k) f(x) d x
$$

donde $K(x, k)$ es el Kernel o núcleo de la transformación. El operador $\mathcal{I}$ es denominado transformación integral, $F(k)$ es la imagen por el operador $\mathcal{I}$ de la función objetivo $f(x)$ y $k$ es la variable de transformación.

Existe una amplia variedad de importantes transformaciones integrales dentro de las cuales se destacan las transformadas de: Laplace y Mellin, cada una de las cuales se define mediante la selección particular del núcleo $K(x, k)$, y diferentes valores de $a$ y $b$. En las siguientes secciones se expondrá la aplicación de la transformada de Mellin y Laplace en la resolución del problema de valoración de activos contingente mediante la aplicación de este tipo de transformaciones sobre la EDP correspondiente. 
Es evidente que el operador $\mathcal{I}$ es lineal, y con el ánimo de obtener $f(x)$ a partir de $F(k)=\mathcal{I}\{f(x)\}$, se introduce el operador inverso $\mathcal{I}^{-1}$ tal que:

$$
\mathcal{I}^{-1}\{F(k)\}=f(x)
$$

que también resulta ser lineal.

\section{Transformada de Mellin}

La transformada integral de Mellin de una función $g(x)$ se puede definir si la función $\left.g(x) x^{k-1} \in L^{1}([0, \infty)]\right)$, es decir si la función $g(x) x^{k-1}$ es integrable sobre los reales no negativos, y la transformada se define como:

$$
g^{*}(k)=\mathcal{M}[g(x)](k)=\int_{0}^{\infty} g(x) x^{k-1} d x \quad R e(k) \leq m
$$

Algunas propiedades de la transformada de Mellin son:

1.

$$
\mathcal{M}\left[x g^{\prime}(x)\right](k)=-k g^{*}(k)
$$

2.

$$
\mathcal{M}\left[x^{2} g^{\prime \prime}(x)\right](k)=\left(k^{2}+k\right) g^{*}(k)
$$

3. La inversa de la transformada de Mellin se define como:

$$
\mathcal{M}^{-1}\left[g^{*}(k)\right](x)=\frac{1}{2 i \pi} \int_{\alpha-i \infty}^{\alpha+i \infty} g^{*}(k) x^{-x} d k \quad \alpha>m
$$




\subsection{Aplicación a la EDP-BS}

Considerando que la función $V\left(t, S_{t}\right)$ es tal que $\frac{\partial V}{\partial t}, \frac{\partial V}{\partial S_{t}}$ y $\frac{\partial^{2} V}{\partial S_{t}^{2}}$ son transformables Mellin, y siendo:

$$
v(t)(k)=\mathcal{M}[V(t, \cdot)](k)=\int_{0}^{\infty} S^{k-1} V(t, S) d S
$$

se puede aplicar la transformada de Mellin a la EDP-BS, obteniendo:

$$
\begin{gathered}
\mathcal{M}\left[\frac{\partial V}{\partial t}\right]=r_{f} \mathcal{M}[V]-\frac{1}{2} \sigma^{2} \mathcal{M}\left[S^{2} \frac{\partial^{2} V}{\partial S^{2}}\right]-r_{f} \mathcal{M}\left[S \frac{\partial V}{\partial S}\right] \\
\frac{d}{d t}(v(t)(k))=-\underbrace{\left(\frac{1}{2} \sigma^{2} k^{2}+\left(\frac{1}{2} \sigma^{2}-r_{f}\right) k-r_{f}\right)}_{p(k)} v(t)(k)
\end{gathered}
$$

de donde,

$$
\frac{d}{d t}(v(t)(k))=-p(k)(v(t)(k)) \quad 0 \leq t \leq T \quad v(T)(k)=f^{*}(k)
$$

que es una ecuación diferencial ordinaria cuya solución es:

$$
v(t)(k)=f^{*}(k) e^{-p(k)}(t-T) \quad 0 \leq t \leq T
$$

y aplicando la transformada inversa sobre (9), con el cambio de variable $k=$ $\alpha+i \tau, d k=i d \tau$, se tiene que:

$$
V(t, S)=\frac{1}{2 \pi} \int_{-\infty}^{\infty} S^{-(\alpha+i \tau)} f^{*}(\alpha+i \tau) e^{\hat{p}(\tau)(t-T)} d \tau
$$

donde, 
$\hat{p}(\tau)=-p(\alpha+i \tau)=\frac{1}{2} \sigma^{2} \tau^{2}+\left[r_{f}-\left(\alpha+\frac{1}{2}\right) \sigma^{2}\right] \tau i-\left[\frac{1}{2} \sigma^{2} \alpha^{2}+\alpha\left(\frac{1}{2} \sigma^{2}-r_{f}\right)-r_{f}\right]$

expresión que permite determinar el valor del derivado en consideración.

\section{Transformada de Laplace}

La transformada de Laplace $\mathcal{L}\{f(x)\}$ se define mediante el núcleo $K(x, k)=$ $e^{-k x}, \operatorname{con} a=0$ y $b=\infty$, luego:

$$
\mathcal{L}\{f(x)\}=\bar{f}(k)=\int_{0}^{\infty} e^{-k x} f(x) d x
$$

y la transformada inversa se define como:

$$
\mathcal{L}^{-1}\{\bar{f}(k)\}=f(x)=\frac{1}{2 \pi i} \int_{c-i \infty}^{c+i \infty} e^{k x} \hat{f}(k) d k \quad c>0
$$

Algunas propiedades relevantes de la transformada de Laplace aplicada sobre las derivadas parciales de una función $u(x, t)$ son:

$$
L\left\{\frac{\partial u}{\partial t}\right\}=k \bar{u}(x, k)-u(x, 0) \quad ; \quad L\left\{\frac{\partial u}{\partial x}\right\}=\frac{d \bar{u}}{d x} \quad ; \quad L\left\{\frac{\partial^{2} u}{\partial x^{2}}\right\}=\frac{d^{2} \bar{u}}{d x^{2}}
$$

\subsection{Aplicación a la EDP-BS}

Al realizar los cambios de variables:

$$
\tau=\frac{\sigma^{2}}{2}(T-t) \quad ; \quad z=\ln \left(S_{t}\right)
$$


se puede considerar una nueva función $f(\tau, x)=V\left(t, S_{t}\right)$, que satisface:

$$
\left\{\begin{array}{l}
\frac{\partial f}{\partial \tau}+\left(\frac{r_{f}}{\sigma^{2} / 2}-1\right) \frac{\partial f}{\partial z}+\frac{\partial^{2} f}{\partial z^{2}}-\frac{r_{f}}{\sigma^{2} / 2} f=0 \\
f(0, z)=V\left(T, e^{z}\right)
\end{array}\right.
$$

y al aplicar la transformada de Laplace sobre (15) se llega a la ecuación diferencial ordinaria de segundo orden:

$$
\frac{d^{2}}{d z^{2}} \bar{f}(k, z)+(m-1) \frac{d}{d z} \bar{f}(k, z)-(m+k) \bar{f}(k, z)+C
$$

donde $m=r_{f} /\left(\sigma^{2} / 2\right)$.

Para resolver la ecuación (16) de define $\bar{f}(k, z)=\exp (\alpha z) \bar{g}(k, z)$, donde $\alpha=(1-m) / 2$, entonces $\bar{g}(k, z)$ resuelve la ecuación:

$$
\frac{d^{2}}{d z^{2}} \bar{g}(k, z)-(b+k) \bar{g}(k, z)+e^{-\alpha z} C=0
$$

donde $b=\alpha^{2}+m$. La ecuación 17 puede resolverse separando las regiones $z>k$ y $z \leq k$, para tener:

$$
\bar{g}(k, z)= \begin{cases}\frac{e^{-(\alpha-1) z}}{k}-\frac{e^{-(\alpha z-k)}}{k+m}+h_{1}(k, z) A_{1}+h_{2}(k, z) A_{2} & \text { si } z>k \\ h_{1}(k, z) B_{1}+h_{2}(k, z) B_{2} & \text { si } z \leq k\end{cases}
$$

con $h_{1}(\kappa, z)=e^{-\sqrt{b+k z}}, h_{2}(\kappa, z)=e^{\sqrt{b+k z}}$ y las constantes $A_{1}, A_{2}, B_{1}$ y $B_{2}$ son determinadas por las condiciones de frontera del problema.

\section{Referencias}

Flajolet, P., Régnier, M., \& Sedgewick, R. (1985). Some uses of the Mellin integral transform in the analysis of algorithm (pp. 241-254). Springer Berlin Heidelberg. 
Frontczak, R., \& Schöbel, R. (2010). On modified Mellin transforms, Gauss-Laguerre quadrature, and the valuation of American call options. Journal of computational and applied mathematics, 234(5), 1559-1571.

Frontczak, R., \& Schöbel, R. (2008). Pricing American options with Mellin transforms (No. 319). Tübinger Diskussionsbeitrag.

Fu, M. C., Madan, D. B., \& Wang, T. (1999). Pricing continuous Asian options: a comparison of Monte Carlo and Laplace transform inversion methods. Journal of Computational Finance, 2(2), 49-74.

Panini, R., \& Srivastav, R. P. (2004). Option pricing with Mellin transnforms. Mathematical and Computer Modelling, 40(1), 43-56.

Sepp, A. (2004). Analytical pricing of double-barrier options under a double-exponential jump diffusion process: applications of Laplace transform. International Journal of Theoretical and Applied Finance, 7(02), 151-175. 